\title{
Extraction of Farey Sequence from Stern-Brocot Sequence
}

\section{S.Deepa , A.Gnanam}

\begin{abstract}
In this paper we define and discuss the properties of Farey Sequence and Stern - Brocot Tree and the principal aim of this paper is to extract Farey sequence from Stern Brocot Tree.
\end{abstract}

Key words: Stern - Brocot Sequence, Farey Sequence, Fibonacci sequence.

\section{INTRODUCTION}

The Stern-Brocot tree has received much attention recently due to its deep connections with physical chemistry [6]. The Stern - Brocot tree was discovered by Moritz Stern [1] in 1858 and Achille Brocot [2] in 1861.It was originally used by Brocot to design gear systems with a gear ratio close to some desired value(like the numbers of seconds in a day) by finding a ratio of smooth numbers (number that decompose into small prime factors)near that value. Since smooth numbers factor into small primes, several small gears could be connected in sequence to generate an effective ratio of the product of their teeth, thus creating a relative small gear train while minimizing its error[3].

The Stern - Brocot tree begins with the numbers $\frac{0}{1}$ and $\frac{1}{0}$. The proceeding levels of the tree are formed by including the Mediant fraction $\frac{a+c}{b+d}$ between every pair of neighbours values $\frac{a}{b}$ and $\frac{c}{d}$ and the procedure is repeated to infinity. Retracing the tree upward gives a series of progressively worse rational approximations with decreasing denominators. Here we try to extract the Farey sequence from the Stern-Brocot tree [3].

\section{Definition 1. (Stern-Brocot Sequence).[7]}

With $s_{0,1}=0$ and $s_{0,2}=1$, we define for $n \geq 0$, $\boldsymbol{S}_{n}=\left\{\boldsymbol{s}_{n, 1}, \boldsymbol{s}_{n, 2}, \boldsymbol{s}_{n, 3} \ldots \ldots \ldots \ldots \ldots . . . . . . \boldsymbol{s}_{n, 2^{n}+1}\right\}$ as the sequence for which, for $k \geq 1, n>0$, $S_{n, 2 k-1}=s_{n-1, k}$ and $s_{n, 2 k}=s_{n-1, k}+s_{n-1, k+1}$ Similarly, with, $q_{0,1}=1, q_{0,2}=0$, we define $Q_{n}$. Then the sequence defined by

$\boldsymbol{H}_{\boldsymbol{n}}=\left\{\boldsymbol{h}_{\boldsymbol{n}, \mathbf{1}}, \boldsymbol{h}_{\boldsymbol{n}, 2}, \boldsymbol{h}_{\boldsymbol{n}, 3} \ldots \ldots \ldots \ldots \boldsymbol{h}_{\boldsymbol{n}, \mathbf{2}^{\boldsymbol{n}}+\mathbf{1}}\right\}$ where $h_{n, i}=\frac{s_{n, i}}{q_{n, i}}$ is called the Stern-Brocot Sequence of order n. It represents the sequence containing both the first $n$ generations of mediants based on $H_{0}$, and the terms of $H_{0}$ itself. [6]

Definition 2. (Parents) [7]

We call $h_{n-1, k}$ and $h_{n-1, k+1}$, the left and right parents respectively of $h_{n, 2 k}$

Defintion 3 (Levels of the Stern- Brocot Tree)[7]

Let $H_{0}$ be level 0 of the Stern- Brocot Tree. For $n>0$, level $n$ of the Stern-Brocot tree is defined as med $H_{n-1}$ where [7]

$\operatorname{Med} H_{n-1}=\left\langle\left(h_{n-1,1} \oplus h_{n-1,2}\right),\left(h_{n-1,2} \oplus\right.\right.$

$h n-1,3 . . . . . . . . h n-1,2 n-1 \oplus h n-1,2 n-1+1$

and $\oplus$ is the child's addition operator where by $\frac{a}{b} \oplus \frac{c}{d}=$ $\frac{a+c}{b+d}$

Level $0 \frac{0}{1} \frac{0}{1}$

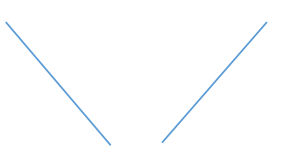

Level $1 \frac{1}{1}$

Level $2 \frac{1}{2} \frac{2}{1}$

Level $3 \frac{1}{3} \frac{2}{3} \frac{3}{2} \frac{3}{1}$

Level $4 \quad \frac{1}{4} \frac{2}{5} \frac{3}{5} \frac{3}{4} \frac{4}{3} \frac{5}{3} \frac{5}{2} \frac{4}{1}$

Level $5 \quad \frac{1}{5} \frac{2}{7} \frac{3}{8} \frac{3}{7} \frac{4}{7} \frac{5}{8} \frac{5}{7} \frac{4}{5} \frac{5}{4} \frac{7}{5} \frac{8}{5} \frac{7}{4} \frac{7}{3} \frac{8}{3} \frac{7}{2} \frac{5}{1}$

Figure1:Levels 0 to 5 of the Stern-Brocot tree

\section{HISTORY OF THE FAREY SEQUENCE[4]}

The Farey Sequene is a pattern that has its origin in quite common numbers[4]. The Farey fractions can be found in all sorts of different applications. The Farey sequence was so named for British born geologist, John Farey (17661826)[4]. In 1816 Farey wrote about the "curious nature of vulgar fractions" in the publication Philosophical Magazine. Given a sequence $\left(F_{N}\right)$ are made up of fractions in lowest terms where the denominator is less than or equal a number $\mathrm{N}$. When the fractions of $F_{1}$ are added together from the mediant property, $\frac{0}{1} \oplus \frac{1}{1}=\frac{1}{2}$, a new fractions falls between the original two is generated[4]. 
This fraction is called the mediant. The next series is found by adding the first two fraction of $F_{2}$ to find the median,$\frac{0}{1} \oplus \frac{1}{2}=\frac{1}{3}[4]$. One finds the mediant of the last two fractions in $F_{2}, \frac{1}{2} \oplus \frac{1}{1}=\frac{2}{3}$. [4] This procedure of finding the mediant between each pair of fraction in the previous Farey sequence is repeated to find the next sequence.[4] The Farey fractions lie in [0,1].[4]

\subsection{Farey Sequence[5]}

The Farey sequence $F_{n}$ for any positive integers ' $n$ ' is the set of irreducible rational numbers $\frac{a}{b}$ with $0 \leq a \leq b \leq n$ and $(a, b)=1$ arranged in increasing order.[5] The first few are

$$
\begin{gathered}
F_{1}=\left\{\frac{0}{1}, \frac{1}{1}\right\} \\
F_{2}=\left\{\frac{0}{1}, \frac{1}{2}, \frac{1}{1}\right\} \\
F_{3}=\left\{\frac{0}{1}, \frac{1}{3}, \frac{1}{2}, \frac{2}{3}, \frac{1}{1}\right\} \\
F_{4}=\left\{\frac{0}{1}, \frac{1}{4}, \frac{1}{3}, \frac{1}{2}, \frac{2}{3}, \frac{3}{4}, \frac{1}{1}\right\} \\
F_{5}=\left\{\frac{0}{1}, \frac{1}{5}, \frac{1}{4}, \frac{1}{3}, \frac{2}{5}, \frac{1}{2}, \frac{3}{5}, \frac{2}{3}, \frac{3}{4}, \frac{4}{5}, \frac{1}{1}\right\}[5]
\end{gathered}
$$

\subsection{Properties of Farey Sequence [9]}

If we have two fractions $\mathrm{a} / \mathrm{b}$ and $\mathrm{c} / \mathrm{d}$ with the properties that $\frac{a}{b}<\frac{c}{d}$ and $|b c-a d|=1$ then the fractions are known as Farey neighbours they appear next to each other in some Farey sequence. The median or Freshman's sum of these two fractions is given by $\left(\frac{a}{b} \bigoplus_{d}^{c}\right)=\frac{a+c}{b+d}$

\section{FIBONACCI SEQUENCE [8]}

The Fibonacci sequence is a set of numbers that starts with a one or a zero followed by a one,and proceeds based on the rule that each number (called a Fabonacci number) is eequal to the sum of the preceding two numbers. [8]

If the Fibanacci sequence is denoted $F(n)$ where $\mathrm{n}$ is the first term in thesequence the following equation obtains for $n=0$ where firsttwo terms are defined as 0 and 1 by convention [8]

$\mathrm{F}(0)=0,1,1,2,3,5,8,13,21,34, \ldots \ldots$

In some case it also followed as

$$
\mathrm{F}(1)=1,1,2,3,5,8,13,21,34 \ldots \ldots
$$

\section{EXTRACTION OF FAREY SEQUENCE FROM STERN- BROCOT SEQUENCE}

The extraction of Farey Sequence from Stern-Brocot sequence is obtained by the following procedure

Let us assume the following

- $I_{n}$ denotes the $n^{t h}$ level of the SternBrocot Sequence. That is $I_{1}=\left\{\frac{0}{1}, \frac{1}{0}\right\}$, $I_{2}=\left\{\frac{0}{1}, \frac{1}{1}, \frac{1}{0}\right\}$ etc.

- $\quad F_{n}$ denotes the $n^{\text {th }}$ level of the Ferey Sequence.

- $\gamma\left(I_{n}\right)$ denoted the $n^{\text {th }}$ term of the Fibonacci sequence and Fibonacci
Sequence terms starts from $n=$ $0,1,2,3,4,5, \ldots$

- Let us define $\gamma\left(I_{0}\right)=0, \gamma\left(I_{1}\right)=$ $1, \gamma\left(I_{2}\right)=1, \gamma\left(I_{3}\right)=2, \gamma\left(I_{4}\right)=3, \gamma\left(I_{5}\right)=$ $5 \ldots . .$. so on. Which follows Fibonacci Sequence from $0,1,1,3,5,8,13 \ldots \ldots$.

- $\quad \gamma\left(I_{n-1}\right)$ denoted the $(n-1)^{t h}$ term of the Fibonacci sequence.

- Always starting values of $i$ in the union should be smaller than the ending value $\gamma\left(I_{n}\right)$ otherwise omit the corresponding unions.

- Omitting the fractions which have common multiples from both numerator and denominator in the sequence.

- Ie., Considering only the fractions whose numerator and denominator in the sequence are Co-primes in the unions.

General Case $1:(n=1,2,3,4,5 \ldots \ldots$

$$
\begin{aligned}
F_{n}=\left\{I_{n+1}\right\}-\left[\left\{U_{i=1}^{\gamma\left(I_{n+1}\right)}\left(U_{k=i-1}^{\gamma\left(I_{n+1}\right)}\left[\frac{k+1}{(k+1)-i}\right]\right)\right\} U\right. \\
i=1 \gamma(I n) k=n \gamma(I n+1) i+1
\end{aligned}
$$

$4+1$

Also be written as
Where $\gamma\left(I_{n}\right)=\frac{1}{\sqrt{5}}\left[\frac{1+\sqrt{5}}{2}\right]^{n+1}-\frac{1}{\sqrt{5}}\left[\frac{1-\sqrt{5}}{2}\right]^{n+1}$ follows the Fibonacci sequence terms starts from $\mathrm{n}=0$.

Iteration 1: $(\mathbf{n}=\mathbf{4})$

We have from General Case

$F_{4}=\left\{I_{4+1}\right\}-$

$\left[\left\{\bigcup_{i=1}^{\gamma\left(I_{5}\right)}\left(U_{k=i-1}^{\gamma\left(I_{5}\right)}\left[\frac{k+1}{(k+1)-i}\right]\right)\right\} U\right.$

$k+1$ $i=1 \gamma(15) k=4 \gamma(15) i+1$

$F_{4}=\left\{I_{5}\right\}-$

$\left[\left\{\mathrm{U}_{i=1}^{5}\left(\mathrm{U}_{k=i-1}^{5}\left[\frac{k+1}{(k+1)-i}\right]\right)\right\} \mathrm{U}\right.$ $i=15 k=45 i+1 k+1$

$F_{4}=\left\{\frac{0}{1}, \frac{1}{4}, \frac{1}{3}, \frac{2}{5}, \frac{1}{2}, \frac{3}{5}, \frac{2}{3}, \frac{3}{4}, \frac{1}{1}, \frac{4}{3}, \frac{3}{2}, \frac{5}{3}, \frac{2}{1}, \frac{5}{2}, \frac{3}{1}, \frac{4}{1}, \frac{1}{0}\right\}-$ $\left\{\frac{1}{0}, \frac{2}{1}, \frac{3}{2}, \frac{4}{3}, \frac{5}{4}, \frac{6}{5}, \frac{2}{0}, \frac{3}{1}, \frac{5}{3}, \frac{4}{1}, \frac{5}{2}, \frac{5}{1}, \frac{6}{1}\right\}$

$F_{4}=\left\{\frac{0}{1}, \frac{1}{3}, \frac{1}{2}, \frac{2}{3}, \frac{1}{1}, \frac{1}{4}, \frac{3}{4}\right\}$ 
Formula Satisfied under Mathematical Induction Method:

$$
\begin{aligned}
F_{n}=\left\{I_{n+1}\right\}-\left[\left\{U_{i=1}^{\gamma\left(I_{n+1}\right)}\left(U_{k=i-1}^{\gamma\left(I_{n+1}\right)}\left[\frac{k+1}{(k+1)-i}\right]\right)\right\} U\right. \\
\\
i=1 \gamma(\operatorname{In}) k=n \gamma(\operatorname{In}+1) i+1
\end{aligned}
$$

$$
\boldsymbol{k}_{i e}+\boldsymbol{I}_{\boldsymbol{n}}=\left\{I_{n+1}\right\}-
$$

$\left\{\begin{array}{c}{\left[\left(\bigcup_{i=1}^{\gamma\left(I_{n}\right)} \bigcup_{k=i-1}^{\gamma\left(I_{n}\right)+\gamma\left(I_{n-1}\right)}\left[\frac{k+1}{(k+1)-i}\right]\right) \bigcup\left(\bigcup_{i=\gamma\left(I_{n}\right)+1}^{\gamma\left(I_{n}\right)+\gamma\left(I_{n-1}\right)} \bigcup_{k=i-1}^{\gamma\left(I_{n}\right)+\gamma\left(I_{n-1}\right)}\left[\frac{k+1}{(k+1)-i}\right]\right)\right]} \\ \bigcup\left[\left(\bigcup_{i=1}^{\gamma\left(I_{n-1}\right)} \gamma \bigcup_{k=n}^{\gamma\left(I_{n}\right)+\gamma\left(I_{n-1}\right)}\left[\frac{i+1}{k+1}\right]\right) \bigcup\left(\bigcup_{i=\gamma\left(I_{n-1}\right)+1}^{i\left(I_{n-2}\right)} \bigcup_{k=n}^{\gamma\left(I_{n}\right)+\gamma\left(I_{n-1}\right)}\left[\frac{i+1}{k+1}\right]\right)\right]\end{array}\right\}$

The Mathematical Induction method was applied only on the dynamic portion of the formula (that is only for union portions by keeping $I_{n+1}$ as the statics position.

Let us prove Mathematical Induction for

$\left[\left\{\cup_{i=1}^{\gamma\left(I_{n+1}\right)}\left(\cup_{k=i-1}^{\gamma\left(I_{n+1}\right)}\left[\frac{k+1}{(k+1)-i}\right]\right)\right\} \cup\left\{\cup_{i=1}^{\gamma\left(I_{n}\right)}\left(\cup_{k=n}^{\gamma\left(I_{n+1}\right)}\left[\frac{i+1}{k+1}\right]\right)\right\}\right]=$

$\left\{\begin{array}{c}{\left[\left(\bigcup_{i=1}^{\gamma\left(I_{n}\right)} \bigcup_{k=i-1}^{\gamma\left(I_{n}\right)+\gamma\left(I_{n-1}\right)}\left[\frac{k+1}{(k+1)-i}\right]\right) \bigcup\left(\bigcup_{i=\gamma\left(I_{n}\right)+1}^{\gamma\left(I_{n}\right)+\gamma\left(I_{n-1}\right)} \bigcup_{k=i-1}^{\gamma\left(I_{n}\right)+\gamma\left(I_{n-1}\right)}\left[\frac{k+1}{(k+1)-i}\right]\right)\right]} \\ \left.\bigcup\left[\left(\bigcup_{i=1}^{\gamma\left(I_{n-1}\right) \gamma\left(I_{n}\right)+\gamma\left(I_{n-1}\right)} \bigcup_{k=n}^{i\left(I_{n-1}\right)+\gamma\left(I_{n-2}\right)}\left[\frac{i+1}{k+1}\right]\right) \bigcup\left(\bigcup_{i=\gamma\left(I_{n-1}\right)+\gamma}^{i\left(I_{n-1}\right)} \bigcup_{k=n}^{i+1}\right]\right)\right]\end{array}\right\}$

Let us prove for $\mathbf{n}=\mathbf{3}$

L.H.S.,

$\left[\left\{\bigcup_{i=1}^{\gamma\left(I_{n+1}\right)}\left(\bigcup_{k=i-1}^{\gamma\left(I_{n+1}\right)}\left[\frac{k+1}{(k+1)-i}\right]\right)\right\} \cup\left\{\bigcup_{i=1}^{\gamma\left(I_{n}\right)}\left(\bigcup_{k=n}^{\gamma\left(I_{n+1}\right)}\left[\frac{i+1}{k+1}\right]\right)\right\}\right]$

$=\left[\left\{\mathrm{U}_{\mathrm{i}=1}^{\gamma\left(\mathrm{I}_{4}\right)}\left(\mathrm{U}_{\mathrm{k}=\mathrm{i}-1}^{\gamma\left(\mathrm{I}_{4}\right)}\left[\frac{\mathrm{k}+1}{(\mathrm{k}+1)-\mathrm{i}}\right]\right)\right\} \cup\left\{\mathrm{U}_{\mathrm{i}=1}^{\gamma\left(\mathrm{I}_{3}\right)}\left(\mathrm{U}_{\mathrm{k}=3}^{\gamma\left(\mathrm{I}_{4}\right)}\left[\frac{\mathrm{i}+1}{\mathrm{k}+1}\right]\right)\right\}\right]$

$=\left[\left\{\cup_{i=1}^{3}\left(\cup_{k=i-1}^{3}\left[\frac{k+1}{(k+1)-i}\right]\right)\right\} \cup\left\{\cup_{i=1}^{2}\left(\cup_{k=3}^{3}\left[\frac{i+1}{k+1}\right]\right)\right\}\right]$

$=\left\{\frac{1}{0}, \frac{2}{1}, \frac{3}{2}, \frac{4}{3}, \frac{2}{0}, \frac{3}{1}, \frac{4}{2}, \frac{3}{0}, \frac{4}{1}, \frac{2}{4}, \frac{3}{4}\right\}$

\section{R.H.S.,}

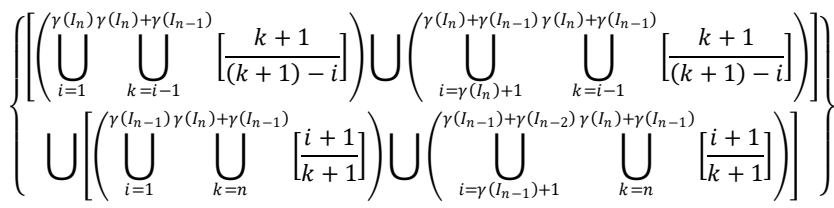
$=\left\{\begin{array}{c}{\left[\left(\bigcup_{i=1}^{\gamma\left(I_{3}\right)} \bigcup_{k=i-1}^{\gamma\left(I_{3}\right)+\gamma(2)}\left[\frac{k+1}{(k+1)-i}\right]\right) \bigcup\left(\bigcup_{i=\gamma\left(I_{3}\right)+1}^{\gamma\left(I_{3}\right)+\gamma\left(I_{2}\right)} \bigcup_{k=i-1}^{\gamma\left(I_{3}\right)+\gamma\left(I_{2}\right)}\left[\frac{k+1}{(k+1)-i}\right]\right)\right]} \\ \bigcup\left[\left(\bigcup_{i=1}^{\gamma\left(I_{2}\right)} \bigcup_{k=3}^{\gamma\left(I_{3}\right)+\gamma\left(I_{2}\right)}\left[\frac{i+1}{k+1}\right]\right) \bigcup\left(\bigcup_{i=\gamma\left(I_{2}\right)+1}^{\gamma\left(I_{2}\right)+\gamma\left(I_{1}\right)} \bigcup_{k=3}^{\gamma\left(I_{3}\right)+\gamma\left(I_{2}\right)}\left[\frac{i+1}{k+1}\right]\right)\right]\end{array}\right\}$ $=\left\{\begin{array}{c}{\left[\left(\bigcup_{i=1}^{2} \bigcup_{k=i-1}^{2+1}\left[\frac{k+1}{(k+1)-i}\right]\right) \bigcup\left(\bigcup_{i=2+1}^{2+1} \bigcup_{k=i-1}^{2+1}\left[\frac{k+1}{(k+1)-i}\right]\right)\right]} \\ \bigcup\left[\left(\bigcup_{i=1}^{1} \bigcup_{k=3}^{2+1}\left[\frac{i+1}{k+1}\right]\right) \bigcup\left(\bigcup_{i=1+1}^{2+1} \bigcup_{k=3}^{2+1}\left[\frac{i+1}{k+1}\right]\right)\right]\end{array}\right\}$

$$
\begin{aligned}
& =\left\{\begin{array}{c}
{\left[\left(\bigcup_{i=1}^{2} \bigcup_{k=i-1}^{3}\left[\frac{k+1}{(k+1)-i}\right]\right) \bigcup\left(\bigcup_{i=3}^{3} \bigcup_{k=i-1}^{3}\left[\frac{k+1}{(k+1)-i}\right]\right)\right]} \\
\bigcup\left[\left(\bigcup_{i=1}^{3} \bigcup_{k=3}^{3}\left[\frac{i+1}{k+1}\right]\right) \bigcup\left(\bigcup_{i=2}^{2} \bigcup_{k=3}\left[\frac{i+1}{k+1}\right]\right)\right]
\end{array}\right\} \\
& =\left\{\frac{1}{0}, \frac{2}{1}, \frac{3}{2}, \frac{4}{3}, \frac{2}{0}, \frac{3}{1}, \frac{4}{2}, \frac{3}{0}, \frac{4}{1}, \frac{2}{4}, \frac{3}{4}\right\}
\end{aligned}
$$

From (1) and (2) formula is true for $n=3$

Assume that formula is true for $n=m$

$\left[\left\{\bigcup_{i=1}^{\gamma\left(I_{m+1}\right)}\left(\cup_{k=i-1}^{\gamma\left(I_{m+1}\right)}\left[\frac{k+1}{(k+1)-i}\right]\right)\right\} \cup\left\{\bigcup_{i=1}^{\gamma\left(I_{m}\right)}\left(\cup_{k=m}^{\gamma\left(I_{m+1}\right)}\left[\frac{i+1}{k+1}\right]\right)\right\}\right]=$

$\left\{\begin{array}{c}\left(\left[\bigcup_{i=1}^{\gamma\left(I_{m}\right)} \bigcup_{k=i-1}^{\gamma\left(I_{m}\right)+\gamma\left(I_{m-1}\right)}\left[\frac{k+1}{(k+1)-i}\right]\right) \bigcup\left(\bigcup_{i=\gamma\left(I_{m}\right)+1}^{\gamma\left(I_{m}\right)+\gamma\left(I_{m-1}\right)} \bigcup_{k=i-1}^{\gamma\left(I_{m}\right)+\gamma\left(I_{m-1}\right)}\left[\frac{k+1}{(k+1)-i}\right]\right)\right] \\ \bigcup\left[\left(\bigcup_{i=1}^{\gamma\left(I_{m-1}\right)} \bigcup_{k=m}^{\left(I_{m}\right)+\gamma\left(I_{m-1}\right)}\left[\frac{i+1}{k+1}\right]\right) \bigcup\left(\bigcup_{i=\gamma\left(I_{m-1}\right)+1}^{\gamma\left(I_{m-1}\right)+\gamma\left(I_{m-2}\right)} \bigcup_{k=m}^{\gamma\left(I_{m}\right)+\gamma\left(I_{m-1}\right)}\left[\frac{i+1}{k+1}\right]\right)\right]\end{array}\right\}$

Now, let us prove for $\mathbf{n}=\mathbf{m}+\mathbf{1}$

$\left[\left\{\cup_{i=1}^{\gamma\left(I_{m+2}\right)}\left(\cup_{k=i-1}^{\gamma\left(I_{m+2}\right)}\left[\frac{k+1}{(k+1)-i}\right]\right)\right\} \cup\left\{\cup_{i=1}^{\gamma\left(I_{m+1}\right)}\left(\cup_{k=m+1}^{\gamma\left(I_{m+2}\right)}\left[\frac{i+1}{k+1}\right]\right)\right\}\right]=$

$\left\{\begin{array}{c}{\left[\left(\bigcup_{i=1}^{\gamma\left(I_{m+1}\right)} \bigcup_{k=i-1}^{\gamma\left(I_{m+1}\right)+\gamma\left(I_{m}\right)}\left[\frac{k+1}{(k+1)-i}\right]\right) \bigcup\left(\bigcup_{i=\gamma\left(I_{m+1}\right)+1}^{\gamma\left(I_{m+1}\right)+\gamma\left(I_{m}\right)} \bigcup_{k=i-1}^{\gamma\left(I_{m+1}\right)+\gamma\left(I_{m}\right)}\left[\frac{k+1}{(k+1)-i}\right]\right)\right]} \\ \bigcup\left[\left(\bigcup_{i=1}^{\gamma\left(I_{m}\right)} \bigcup_{k=m+1}^{\gamma\left(I_{m+1}\right)+\gamma\left(I_{m}\right)}\left[\frac{i+1}{k+1}\right]\right) \bigcup\left(\bigcup_{i=\gamma\left(I_{m}\right)+1}^{\gamma\left(I_{m}\right)+\gamma\left(I_{m-1}\right)} \bigcup_{k=m+1}^{\gamma\left(I_{m+1}\right)+\gamma\left(I_{m}\right)}\left[\frac{i+1}{k+1}\right]\right)\right]\end{array}\right\}$

Consider,

$$
\begin{aligned}
& {\left[\left\{\bigcup_{i=1}^{\gamma\left(I_{m+2}\right)}\left(\bigcup_{k=i-1}^{\gamma\left(I_{m+2}\right)}\left[\frac{k+1}{(k+1)-i}\right]\right)\right\}\left\{\bigcup_{i=1}^{\gamma\left(I_{m+1}\right)}\left(\bigcup_{k=m+1}^{\gamma\left(I_{m+2}\right)}\left[\frac{i+1}{k+1}\right]\right)\right\}\right] } \\
= & {\left[\left\{U_{i=1}^{\gamma\left(I_{m+1}\right)+\gamma\left(I_{m}\right)}\left(\cup_{k=i-1}^{\gamma\left(I_{m+1}\right)+\gamma\left(I_{m}\right)}\left[\frac{k+1}{(k+1)-i}\right]\right)\right\} \cup\right.} \\
\{ & \left.\left\{U_{i=1}^{\gamma\left(I_{m}\right)+\gamma\left(I_{m-1}\right)}\left(\cup_{k=m+1}^{\gamma\left(I_{m+1}\right)+\gamma\left(I_{m}\right)}\left[\frac{i+1}{k+1}\right]\right)\right\}\right] \\
= & {\left[\left\{U_{i=1}^{\gamma\left(I_{m+1}\right)} U_{\gamma\left(I_{m+1}\right)+1}^{\gamma\left(I_{m+1}\right)+\gamma\left(I_{m}\right)}\left(U_{k=i-1}^{\gamma\left(I_{m+1}\right)+\gamma\left(I_{m}\right)}\left[\frac{k+1}{(k+1)-i}\right]\right)\right\} \cup\right.}
\end{aligned}
$$

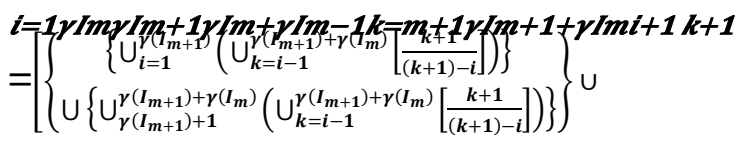

\section{$i=1 \gamma / m k=m+1 \gamma / m+1+\gamma I m i+1$}

$k+1 i=\gamma I m+1 \gamma I m+\gamma I m-1 k=m+1 \gamma I m+1+\gamma I m i+1 k+1$

Hence it is true for $n=m+1$.

Thus second dynamic portion of the formula was derived under Mathematical Induction method which is valid for $(\mathbf{n}=\mathbf{3}, \mathbf{4} \ldots . . .$.$) by keeping \boldsymbol{I}_{\boldsymbol{n}+\mathbf{1}}$ as constant.

Therefore in general,

$\boldsymbol{F}_{\boldsymbol{n}}=\left\{\boldsymbol{I}_{\boldsymbol{n}+\mathbf{1}}\right\}-$ 
$\left\{\begin{array}{c}{\left[\left(\bigcup_{i=1}^{\gamma\left(I_{n}\right)} \bigcup_{k=i-1}^{\gamma\left(I_{n}\right)+\gamma\left(I_{n-1}\right)}\left[\frac{k+1}{(k+1)-i}\right]\right) \bigcup\left(\bigcup_{i=\gamma\left(I_{n}\right)+1}^{\gamma\left(I_{n}\right)+\gamma\left(I_{n-1}\right)} \bigcup_{k=i-1}^{\gamma\left(I_{n}\right)+\gamma\left(I_{n-1}\right)}\left[\frac{k+1}{(k+1)-i}\right]\right)\right]} \\ \bigcup\left[\left(\bigcup_{i=1}^{\gamma\left(I_{n-1}\right)} \bigcup_{k=n}^{\gamma\left(I_{n}\right)+\gamma\left(I_{n-1}\right)}\left[\frac{i+1}{k+1}\right]\right) \bigcup\left(\bigcup_{i=\gamma\left(I_{n-1}\right)+1}^{\gamma\left(I_{n-1}\right)+\gamma\left(I_{n-2}\right)} \bigcup_{k=n}^{\gamma\left(I_{n}\right)+\gamma\left(I_{n-1}\right)}\left[\frac{i+1}{k+1}\right]\right)\right]\end{array}\right\}$

\section{General Case (2)}

The alternate way of extracting the Farey Sequence from the Stern- Brocot sequence is given by

$$
F_{n}=\left\{I_{n} \cup \frac{1}{n} \cup \frac{n-1}{n}\right\}-\left[\left\{U_{i=1}^{\gamma\left(I_{n-1}\right)}\left(\cup_{k=i-1}^{\gamma\left(I_{n}\right)}\left[\frac{k+1}{(k+1)-i}\right]\right)\right\} \cup\right.
$$

Where $\gamma\left(I_{n}\right)=\frac{1}{\sqrt{5}}\left[\frac{1+\sqrt{5}}{2}\right]^{n+1}-\frac{1}{\sqrt{5}}\left[\frac{1-\sqrt{5}}{2}\right]^{n+1}$ follows the Fibonacci sequence terms.

\section{CONCLUSION}

In this paper, definition and properties of Farey sequences and Stern- Brocot sequence have been studied and following result and conclusions were drawn:

- Generation of Farey Sequence from SternBrocot sequence.

- Formula was derived under Mathematical Induction.

\section{REFERENCES}

1. M.A Stern,Ueber eine Zahlentheoretische function,J.Reine Agnew Math 55(1858), 193-220.

2. A.Brocot, Calculdes rouages $\mathrm{Pal}$ approximation,nouvellle method,Revue chronometrique 3 (1861),186-194.

3. B.Hayes,Computing science: on the teeth of wheels, American Scientist 88(2000),296-300.

4. A. Gnanam, C. Dinesh, International Journal of Science and Research (IJSR), Volume 4, Issue 2, December 2016.

5. Weisstein,Eric W. "Farey Sequence" .From MathWorld-A WolFram Web Resource. http://mathworld.Wolfram.Com/Farey Sequence.html.

6. J.G. Freire, J.A.C. Gallas, Stern-Brocot tree in the periodicity of mixed-mode oscillations, Phys. Chem. Chem. Phys. 13 (2011), 12191-12198.

7. The Stern-Brocot Continued Fraction, Bruce Bates School of Mathematics and Applied Statistics, University of Wollongong, Wollongong, NSW, Australia, Publised on $8 / 11 / 14$

8. Whatis.techtarget.com/definition/Fibonacci.sequence.

9. https://www.maths.ed.ac.uk/ v1 ranick/fareyproject.pdf. The Farey Sequence,Jonathan Ainswoth, Michael Dawson, John pianta, James Warwick. Year 4 project,School of Mathematics,University of Edinurgh, March 15,2012. 